\title{
Advances in Tip-Enhanced Spectroscopy from Optical Nano-Crystallography to Ultrafast Spectroscopy
}

\author{
Markus B. Raschke
}

Department of Physics, Department of Chemistry and JILA, University of Colorado at Boulder, Boulder, CO, 80309

The use of ultrasharp scanning probe tips in scattering-type scanning near-field optical microscopy provides a means for all-optical resolution down to 10's of $\mathrm{nm}$. Via plasmonic noble metal tips a spatially confined and locally enhanced excitation can be generated to perform spectroscopies which would otherwise suffer from weak or limited signal levels. In particular, tip-enhanced Raman scattering (TERS) has shown strong potential due to the capability for chemical fingerprinting and single molecule sensitivity; however, the application of TERS to crystalline systems has not yet been fully explored. Furthermore, the mode mismatch between the direct apex-illumination and the localized apex excitation in conventional Raman scattering limits excitation efficiency and generates a far-field background, often obscuring the TERS signal.

We first demonstrate the extension of TERS to the study of crystalline materials, utilizing the symmetry-selective Raman response to image ferroelectric domains in perovskite $\mathrm{BaTiO}_{3}$ nanorods. Secondly, we will discuss the development and spectroscopic applications of a novel nanoconfined light source achieved by the adiabatic nanofocusing of SPPs into the apex of electrochemically etched $\mathrm{Au}$ TERS tips [1]. This arises from the radius-dependent index of refraction experienced by SPPs propagating along the tip, resulting in the continuous mode-matched transformation of a cylindrical SPP mode into a nanometer localized excitation at the apex of the waveguide. We demonstrate $i$ ) sub-25 nm spatial nanoconfinement achieved by SPP nanofocusing, $i i$ ) backgroundfree TERS with improved sensitivity, and iii) ultrafast nonlinear spectroscopy in combination with pulseshaping for full femtosecond temporal and nanometer spatial control of optical fields.

In order to access the necessary degrees of freedom to probe the crystal symmetry we utilize a sideillumination TERS geometry as illustrated in Fig. 1a) to obtain independent control of polarization, wavevector of the incident and scattered light, and crystallographic orientation. The necessary selection rules to perform optical nano-crystallography are derived as a combination of the crystal Raman tensor, momentum conservation, and the symmetry of the near-field tip scattering geometry. The capabilities are demonstrated on $\mathrm{BaTiO}_{3}$ nanorods, with the shear-force AFM topography of a rod shown in Fig. 1b) and the corresponding spectrally integrated TERS signal in Fig. 1c). From the Raman selection rules we determine the region of high signal on the rod to correspond to a ferroelectric domain as illustrated in Fig. 1d) [2].

Optical nanofocusing is achieved through the grating coupling of SPPs onto electrochemically etched Au tips [3] as illustrated in Fig. 2a). By scanning the tip across an ultrasharp Si step edge we can determine the spatial extent of the nanoconfined SPP to be $<25 \mathrm{~nm}$ (Fig. 2b) [4]. Nanofocusing efficiency of up to $9 \%$ at $800 \mathrm{~nm}$ results in energy concentration into the tip-apex 20 times more efficient than under equivalent direct apex-illumination [5].

Background-free TERS measurements using grating-coupled tips with $632.8 \mathrm{~nm}$ excitation of Malachite Green on a Au substrate are shown in Fig. 2c). The localization of the TERS signal within $10 \mathrm{~nm}$ of the surface shows the high degree of nanoconfinement achieved, while the absence of 
direct tip-apex illumination leads to the elimination of the far-field background that frequently limits the contrast in TERS [5].

Through the combination of femtosecond pulses with pulse shaping we demonstrate the simultaneous and independent control over the spatial and temporal characteristics of optical fields on the nanoscale. Spectral phase and amplitude pulse shaping is used in combination with MIIPS to optimize pulse duration of the apex emission. Using the second-

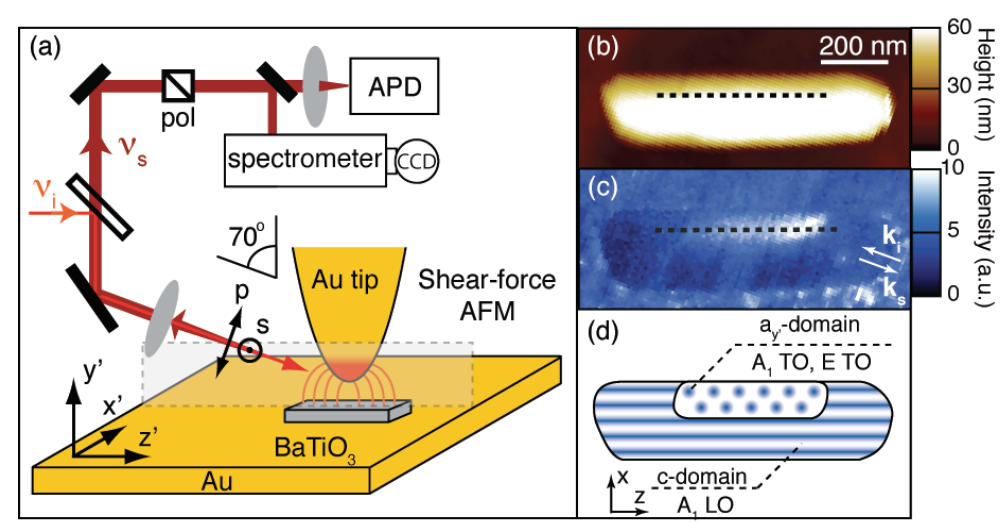

Fig. 1. a) Experimental TERS setup to allow for optical nanocrystallography. b) Shear-force AFM topography and c) corresponding spectrally integrated TERS signal showing a ferroelectric domain as illustrated in d).

harmonic generation (SHG) from the tip apex we perform frequency-resolved optical gating (FROG) measurements of the nanofocused pulses as shown in Fig. 2d). From FROG reconstruction we observe pulses with a duration as short as 16 fs at the tip [Berweger et al., in preparation].

References:

[1] M.I. Stockman, Phys. Rev. Lett., 93, 137404 (2004).

[2] S. Berweger, C.C. Neacsu, Y. Mao, H. Zhou, S.S. Wong, and M.B. Raschke, Nature

Nanotechnol., 4, 496 (2009).

[3] C. Ropers et al., Nano Lett., 7, 2784 (2007).

[4] C.C. Neacsu, S. Berweger, R.L. Olmon, L.V. Saraf, C. Ropers, and M.B. Raschke, Nano Lett., 10, $592(2010)$.

[5] S. Berweger, J.M. Atkin, R.L. Olmon, and M.B. Raschke, J. Phys. Chem. Lett., 1, 3427 (2010).
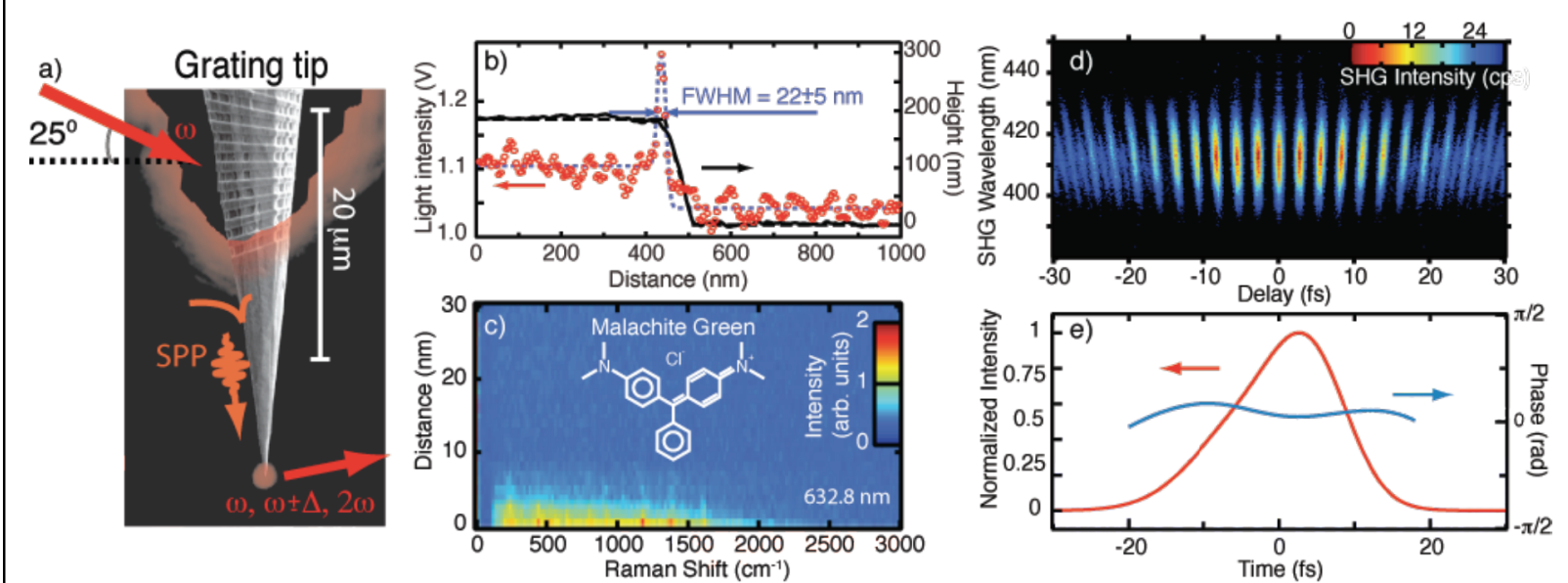

Fig. 2. a) Schematic of the grating-coupling, SPP propagation, and subsequent re-emission of nanofocused excitation. b) Spatial extent of the nanofocused excitation. c) Grating-coupled TERS of a monolayer of Malachite Green on an Au surface. d) FROG measurement acquired with SHG generated at the tip-apex by nanofocused SPPs, demonstrating a pulse duration of $16 \mathrm{fs}$ as determined by e) FROG reconstruction of the temporal profile and phase of the pulse. 